\title{
Cuerpo, escuela y sociedad en la construcción de imagen corporal
}

BODY, SCHOOL AND COMPANY IN THE CONSTRUCTION OF CORPORAL IMAGE

CORPO, ESCOLA E SOCIEDADE NA CONSTRUÇÃO DE IMAGEM CORPORAL

\author{
Marly Stella Pérez Castro* / marperezca@yahoo.es
}

\section{Resumen}

El presente documento destaca, como eje principal, la construcción de la noción de imagen corporal, involucrando temáticas de contexto, ópticas propias, ajenas y mixtas, iniciando con referentes contextuales e históricos a propósito del cuerpo y la forma como se filtra su concepto social y cultural en la escuela. Luego se analiza el tema con vista al interior de la escuela, específicamente del aula, reafirmando que el cuerpo no ignora la fuerza que ejerce, su aprendizaje y las vivencias individuales y colectivas, en la construcción de la imagen. Por último se exponen las reflexiones finales, presumiendo un nivel de comprensión del lector para entender la dinámica fluctuante entre el cuerpo, la escuela y la sociedad, en la construcción de imagen corporal.

\section{Summary}

This paper highlights the construction as the main axis of the concept of body image, involving issues of context, optical own foreign and mixed, starting with contextual reference and historical purpose of the body and how you filter the social and cultural concept the school. Then, we analyze the issue facing the inside of the school, specifically the classroom, reaffirming that the body does not ignore the force of their learning and individual and collective experiences in building the image. Finally, we set the final reflections, assuming a level of understanding of the reader to understand the dynamic fluctuating between body, school and society in the construction of body image.

\section{Resumo}

Este artigo destaca a construção como o eixo principal do conceito de imagem corporal, envolvendo questões de contexto, óptico próprio estrangeiro e misto, começando com referência contextual e objetivo histórica do corpo e como você filtrar o conceito social e cultural da escola. Em seguida, analisamos a questão de frente para o interior da escola, especificamente a sala de aula, reafirmando que o corpo não ignorar a força de seu aprendizado e experiências individuais e coletivas na construção da imagem. Por fim, defina as reflexões finais, assumindo um nível de compreensão do leitor a compreender a dinâmica entre a escola flutuante, corpo e sociedade na construção da imagem corporal.

\section{Palabras clave}

Cuerpo, imagen corporal, corporeidad, sociedad, medios, escuela

Key words

Body, body image, bodily, Society, Media, School

Palavras chave

Corpo, imagem corporal, corporal, sociedade, escola média

\footnotetext{
* Docente de básica secundaria en el Colegio Santa Librada I.E.D., Especialista en Docencia de las Ciencias, Universidad Pedagógica Nacional (2011), estudiante de Maestría en Docencia de las Ciencias Naturales de la Universidad Pedagógica Nacional.
}

Fecha de recepción: 10 de mayo de 2012 / Fecha de aprobación: 8 de junio de 2012 


\section{Introducción}

La adolescencia, época de acné, amigos, amores y rupturas, ha significado también una etapa determinante en la fijación de normas sociales estéticas, particularmente de aquellas convencionales, donde los detalles físicos que apuntan a una exagerada precisión y un estricto moldeamiento corporal parecen tener mayor relevancia. En la afanosa búsqueda por la perfección, aparece de manera casi que imperceptible la imagen corporal, la cual tiene sus sólidos asientos en consideraciones de tipo morfológico y anatómico, pues estos cambios son quizá los más visibles, inmediatos y por qué no decirlo, impactantes; la persona trae consigo modificaciones comportamentales, en su actitud y en el juego de roles sociales asignados.

En esta etapa ocurre frecuentemente una crisis interna del ser, donde el modelo estético reinante choca con la evolución y el desarrollo fisiológico humano individual, enmarcado todo esto en un contexto en el que prevalece el atractivo físico. Poco a poco, sin darnos cuenta, hemos llegado a la construcción de la imagen corporal, la cual empieza en la etapa adolescente, pero a lo largo de toda la vida se afianza, ratifica, moldea y por último, se fija; las alteraciones en la estructuración de dicha imagen son frecuentes, especialmente en el sexo femenino, al punto de convertirse en un "descontento normativo" (Rodin, Silverstein y Striegel-Moore, 1985).

Su constitución no es solo cognitiva, se nutre de valores sociales, tradiciones y patrones culturales, costumbres y valoraciones subjetivas de tipo consumista que permiten establecer categorías y jerarquización de los géneros y entre los géneros. Autores como Bourdieu (1986) señalan que: "las condiciones sociales se inscriben en los cuerpos poniendo, así, de relieve la condición política de dispositivos como la apariencia, el modo de presentarse ante los demás, las marcas corporales, la alimentación, etc.", (Pedraz, M., 2010), constituyéndose en un mecanismo particular de asociación a las normas culturales y a la época.

La educación es decisiva en la asimilación de los patrones culturales para la clasificación social de normas estéticas que inciden en la postura propia de imagen corporal, ya que parte de las funciones de este proceso pedagógico es hacer converger los procesos cognitivos, afectivos y sociales, individual y colectivamente.
Con ello se forja la autopercepción de un individuo, influyendo en su propia vida, muy especialmente en sus preferencias sociales, emocionales y hasta en sus elecciones laborales.

\section{Análisis y ópticas corpóreas}

Imaginemos la siguiente escena: voy a una fiesta y quiero mi mejor atuendo para impresionar a todos los asistentes. Al dirigirme al almacén imagino la ropa, los zapatos y accesorios; entro, miro y escojo según lo planeado; voy al vestier. Por último me miro al espejo y ¡Oh! ¡Sorpresa! ¡Hay alguien en el cristal que no soy yo! (o por lo menos no quien yo quiero ver) ¿Qué pasó? ¿Acaso siento que no conozco mi cuerpo?

Es muy común sentirse confundida cuando una tercera óptica nos refleja tal cual como somos. La situación parece complejizarse cuando nuestra percepción no es igual a nuestro imaginario y que ese imaginario poco o nada tiene que ver con el estereotipo del contexto. ¿Mi cuerpo es lo que soy? Pese a que el concepto de imagen corporal sea muy utilizado en psicología, ciencias y en la educación, no existe una definición concreta y única de ésta, de su evaluación y de las alteraciones de la misma. Esta noción se forma sigilosamente con las normas sociales y culturales de una región determinada y de las formas cambiantes que pida la sociedad.

Paul Schilder, Ilamado el pionero en el análisis multidimensional del concepto de imagen corporal, lo ha definido como: "la figura de nuestro propio cuerpo que formamos en nuestra mente, es decir, la forma en la cual nuestro cuerpo se nos representa a nosotros mismos" (Schilder, 1950). La imagen corporal es influida por nuestra emoción, nuestra personalidad, nuestra sociedad y por esto se expande más allá de los límites del cuerpo. Hay un profundo vínculo entre la imagen corporal propia y la de los demás que incluso alcanza proximidades espaciales y afectivas. Es allí cuando se establecen las identificaciones y proyecciones que permiten que factores internos y externos ${ }^{1}$ configuren el estereotipo reinante.

\footnotetext{
1 Los factores internos están definidos como creencias, prácticas y conductas del individuo, mientras que los externos se refieren a aquellos trasmitidos por el entorno, tales como mensajes audiovisuales, expresiones de los docentes, padres y amigos y todas aquellas organizaciones que resulten significativas para la cultura social de consumo.
} 


\section{Cuerpo y percepción}

Históricamente, los distintos modelos corporales ${ }^{2}$ han legitimado privilegios y restricciones, convirtiéndose en un proyecto que plasma ideologías, a veces dominantes a veces recesivas, aparentemente naturales, siendo un "lugar" de control político, social y económico. Bourdieu (1986), habla de "capital corporal" para dar cuenta de las características corporales que permiten ciertos privilegios en una sociedad. El cuerpo y su desempeño físico constituyen en nuestra sociedad actual un potente referente de identidad y de logros personales, que en otras culturas o épocas anteriores, también fueron relevantes y significaron una estratificación socioeconómica. Por ejemplo, anteriormente una persona con alguna deformidad física en Grecia, era reprobada para desempeñar un cargo público o un estatus social privilegiado, paralelo a la irónica aceptación social por distinguirse en festivales deportivos (Elias, 1986, p. 165).

El cuerpo siempre ha sido importante culturalmente, su percepción dejó de ser ese concepto lineal derivado de órganos y huesos, para convertirse en una noción más compleja que se tatúa simbólicamente; es una representación social, política, económica, pero sobre todo pública. La visión de quienes asumen su cuerpo así, es más compleja y sus manifestaciones sociales ejercen mayor presión en el contexto que la de aquellos que limitan su importancia y lo proveen de una utilidad fisiológica y de protección.

Sumergida en un boom tecnológico y digital, la sociedad actual ha logrado de manera considerable reducir las distancias, los esfuerzos y el contacto físico entre las personas. El Chat, las redes sociales, los mensajes de texto y correos de voz, son algunas formas de comunicación que han hecho clic en nuestra era y que han desplazado otras, donde el cuerpo tiene protagonismo. Sin embargo, estas condiciones no minimizan las valoraciones éticas y estéticas del cuerpo, así como su función comunicativa y representativa social. La fisonomía humana es, y seguirá siendo, la referencia real más cotidiana del ser y sentir; evidencia física de la conciencia misma que invita a mezclar sentimientos y percepciones, y a construir un modelo propio de imagen corporal pública y privada. En términos de D. Denis:

\footnotetext{
2 Lo corporal se ha entendido como reflejo de méritos o vicios: los ojos son el espejo del alma, llevarlo en la sangre $\mathrm{o}$, con verlo me temblaron las piernas. La literatura y las canciones relatan y expresan sentimientos presentándonos ejemplos en lo corporal, pareciendo una especie de lienzo donde se pintan todo tipo de colores.
}

Las elevadas apariencias físicas pasan a ocupar el lugar de las esencias, se configura una nueva escala de valores que condiciona y moldea la percepción de los sujetos, las posibilidades y formas de conocer, sentir y actuar; y así se construye la nueva definición corpórea (Denis, D., 1980).

\section{La sociedad de los medios y su visión corpórea}

La dinámica social y sus prácticas cotidianas han convertido al cuerpo en un terreno donde transitan elementos de consumo de sectores exclusivos de las capas ${ }^{3}$ sociales, que transforman gustos en necesidades y apariencias en costumbres, convirtiendo inevitablemente, el armazón óseo muscular en un vehículo de expresiones y sublimes manifestaciones.

En una mirada panorámica del contexto, se puede ver al cuerpo como una metamorfosis donde se le induce a exhibir elementos que no precisan ser de sí mismo o de su naturaleza. Un claro ejemplo de lo anterior sucede cuando al comercializar un carro o un teléfono, la empresa que lo promociona dispone de mujeres atractivas vestidas sugestivamente, para atraer a un público exclusivo que de manera ingenua cree que adoptará tal imagen al poseer este aparato. Al respecto, Foucault señala que:

No es la primera vez, indudablemente, que el cuerpo constituye el objeto de intereses tan imperiosos y tan apremiantes; en toda sociedad, el cuerpo queda prendido en el interior de poderes muy ceñidos, que le imponen coacciones, interdicciones u obligaciones (Foucault, M., 1978).

Atomizando un poco más la realidad local, vemos individuos que son pancartas móviles, exhibiendo chaquetas con iniciales como GAP o extranjerismos como ¡Just do It! ¿Hay un discurso de poder en ellos que no tenga que ver con lo que el medio y las marcas promocionen y parafraseen, y que al final cada individuo diga interior $y$ exteriormente: Soy Nike, Soy GAP4?

\footnotetext{
3 Se refiere la expresión "capas" y no estrato, pues cubren a los individuos que se encuentran en determinado estatus de manera tal que ellos comulgan sólo con los elementos o condiciones que estén dentro de esta.

4 Empresas multinacionales de este tipo ofrecen campañas publicitarias agresivas para posicionar su nombre, fidelizar al cliente y hacerlo un estilo de vida. Sin embargo, los medios y la sociedad modifican los patrones dependiendo de los estratos y las culturas. Es el caso de las camisetas con el logotipo de GAP, que, con su proliferación comercial, bajos costos y fácil acceso, asumieron en el común de la sociedad local, de estratos bajos, un significado particular y distinto del original, con la frase de Gamín Altamente Peligroso.
} 
Y qué decir de aquellas atribuciones nominales materiales a lo corpóreo como aquel boxeador apodado "mano de piedra" o la destacada mujer inglesa de poder político llamada la "Dama de hierro". Con todo esto sólo se quiere producir una sutil amalgama de condiciones físicas y estereotipos sociales emergentes, que luego genere sensaciones o emociones en las personas a partir de máximas lapidantes.

Los medios audiovisuales y la sociedad orientan su cultura definiéndola como algo uniforme y único, viendo a diario los esfuerzos vanos de individuos por alcanzar el ideal corporal acudiendo a ropas apretadas, dolorosos masajes, cirugías y prótesis..., como lo refiere Silvia Tubert en el texto de Ruth Sandoval: "La preocupación por el estereotipo funciona como una poderosa estrategia de normalización que busca la producción de cuerpos dispuestos a transformarse y mejorar al servicio de las normas sociales (Tubert, S., 2001).

\section{El problema de negación}

¿Esto es lo que soy? ¡Me veo y no me reconozco!, comentarios de este tipo y muchos otros deambulan en el territorio físico demarcado entre un individuo y un espejo o una fotografía. Casi siempre las personas tienden a desconcertarse con lo que una tercera óptica les muestra sin tapujos, reflejando una falsa indiferencia a lo evidente, tomando actitudes de aparente olvido a lo que convive con ellas y que no se puede renunciar sólo con cerrar los ojos o cambiar el tema.

Por el contrario, una persona tiende a acudir a métodos que permitan restituir la autopercepción tenida y que fue desdibujada cuando se enfrentó a una realidad de cristales y retratos. Es claro que ese cuerpo que muestra valor, prestigio y clase, es también objeto de violencia física y emocional por parte de su propietario; y cuando se habla en estos términos, se quiere expresar textualmente las agresiones y abusos a los que es sometido el cuerpo humano, en un afán por interpretar de forma exterior lo que se anhela interiormente.

En esa búsqueda por igualar la percepción propia por aquella reflejada ante los demás, es donde la imagen corporal impera sobre el conocimiento real del organismo y sus funcionalidades. De lo contrario la persona seguiría en ese estado de ignorancia social y no desplazaría su concepción por una que no corresponde con su realidad. Pero, ¿por qué se ha de negar el cuerpo?
Tal parece que la importancia asignada al cuerpo toma como eje modelador a la sexualidad, donde la búsqueda por llamar la atención de forma erótica y despertar todo tipo de sensaciones 5 , se convierte en una de sus funciones principales, al punto de mostrar con el cuerpo la verdadera identidad. Cuando no se logran estos efectos, la persona tiende a lesionarse y hacerse recriminaciones que lo único que buscan es justificar las razones por las cuales no se bajaron los kilos de más o por qué la piel no tomó el color deseado. Una vez más la imagen corporal se va fortaleciendo con connotaciones sociales y percepciones fisiológicas alejadas de lo que es una constitución corpórea. De ahí la frustración experimentada por adolescentes ante un esfuerzo por transformarse en figuras delgadas, y las consecuentes decisiones dañinas, y hasta suicidas, tomadas por algunos jóvenes para alcanzar la imagen corporal de moda (Toro y Vilardell, 1987).

Al negar el cuerpo se está expresando más que la palabra No; de hecho, esa palabra pocas veces es escuchada cuando se manifiesta una inconformidad de este tipo ${ }^{6}$. No sólo se excluyen centímetros de más o de menos, se niega la identidad construida en la mente y reflejada en los músculos y en la piel. Es negar también la forma como me muestro a los demás y asumir de forma resignada la percepción de los demás a partir de sus ópticas. Foucault menciona que: "la sexualidad forma parte de nuestro comportamiento, es un elemento más de nuestra libertad. La sexualidad es obra nuestra, es una creación personal y no la revelación de aspectos secretos de nuestros deseos" (Foucault, M., p. 1978).

Por eso cuando existe un prototipo físico apartado de lo circundante en el contexto, hay en el individuo una crisis de negación a lo construido con sus verdaderos hábitos y costumbres que lo inhiben a mostrarse y a identificarse. No hay una integración entre lo individual y lo social, generando estados depresivos, derrotas internas y la no aceptación físico-emocional de sí mismo.

\footnotetext{
5 En estas sensaciones se incluyen admiración, deseo, placer, envidias y hasta odios por parte del mismo sexo o del sexo opuesto.

6 Recordemos que la ignorancia o la indiferencia son las manifestaciones comunes que un individuo refleja en la sociedad, pero que en su desnudez interior emocional, sí niega el cuerpo.
} 


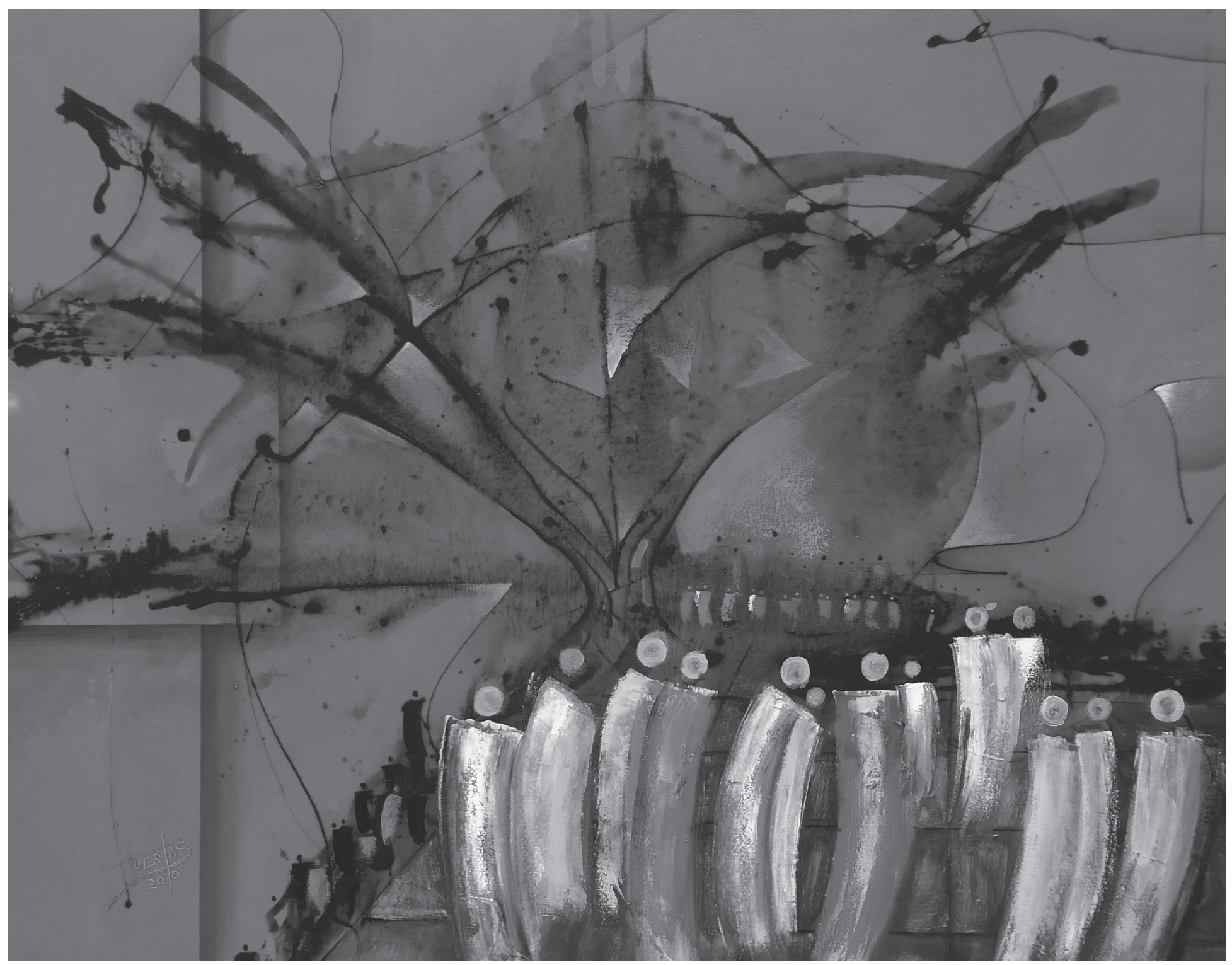

Segundo Arístides Huertas Torres ॥ Título: De la serie Caminantes ॥Técnica: Óleo sobre tela ॥ Dimensiones: $110 \mathrm{~cm}$ x $120 \mathrm{~cm}$ 


\section{El cuerpo como herramienta para el aprendizaje}

El cuerpo pide exhibirse, lucirse, mostrarse y, por momentos, quiere ocultarse. Todo esto es consecuencia de revelar un cuerpo cansado de estar rígido y que se ha soltado de ataduras sociales y escolares; que busca fundamentalmente introducirlo en un cuerpo disciplinado y desinteresado por sí mismo y por sus potencialidades como medio de disfrute, gozo, conocimiento y bienestar.

Cautivo entre pupitres y sillas, a veces con los pies colgados o forzándose intentando encajar en un escritorio, trata de ser silenciado. El aula de clases, incluyendo a su docente, frecuentemente domestica de forma disimulada lo corporal. Más allá de la necesidad de impartir aprendizajes acerca de juicios del ritmo biológico, se debe preguntar si es posible enseñar los cuerpos de las personas sin tener en cuenta los límites invisibles de las asignaturas o las diferencias y complementariedades individuales.

El cuerpo entra en la escuela, pero no logra ajustarse en una sola materia; requiere de todas para configurarse. Parece un gran rompecabezas que se aprecia y se contempla cuando es terminado en todas sus partes; antes sólo es puramente observable. No cabe duda de que la dimensión corporal se enseña desde cualquier área, y las experiencias y vivencias adquiridas se afianzan en el estudiantado y se toman como norma general.

Ahora bien, ocurre otra condición a la que podría Ilamar limitante para enseñar el cuerpo y desde el cuerpo; y no es propiamente el estudiante, su entorno o la asignatura. Es obvio que hay otro tipo de ausencia: la de los cuerpos de los docentes cuando se enfrentan a la enseñanza corpórea. Todo acto comunicativo implica una interacción donde el cuerpo juega un papel destacado. Así como lo describe André Lapierre (1990):

Cuando se habla del cuerpo en la educación, no se trata solamente del cuerpo del estudiante [...] también del cuerpo del educador. Este vínculo frecuentemente es olvidado, pues la relación no es solo maestro-alumno como relación audiovisual o verbal-escritural. Es también una relación psicoafectiva y psicotónica en la que cada uno incluye su personalidad. Querer limitar la labor pedagógica sin modificar las capacidades de relación del educador; su capacidad de escuchar, de comprender y de dominar, simplemente es una ilusión.

El maestro debe lograr una interacción respetuosa con sus alumnos, con el tema y con el contexto ${ }^{7}$. Si esta tríada no es conseguida, nuevamente se caería en el vicio segmentario de la enseñanza del cuerpo. La participación del docente en el juego de roles y en la construcción autónoma de la imagen es fundamental, pues garantiza al estudiante la veracidad de lo que dice y lo que hace. $\mathrm{Si}$ el profesor se limita a instruir y modelar los comportamientos del estudiante frente a la construcción de la imagen corporal, pero no participa ni se compromete en su consecuente significación, está excluyéndose del contexto, de su función social y de su responsabilidad con el aprendizaje, permitiendo perder la fuerza de sus palabras y sus juicios.

El cuerpo no debe limitarse en la enseñanza de su estructura y funcionamiento, sino que debe ser abordado desde su dimensión social y su concepción global que lo orienta a ser parte transversal de un proceso pedagógico. A pesar de que la visión corpórea sea vista sólo desde una óptica fisiológica o de entrenamiento en la escuela, su visión resulta más impositiva culturalmente, al punto que existen modelos corpóreos dominantes, que se han creado más por la tendencia de moda reinante en la época y el lugar, que por la constitución fenotípica o genotípica predominante.

Enseñar el cuerpo humano no sugiere una ruta única, que no permite devolverse o afianzarse en lo aprendido; no es aprender lo orgánico y fisiológico, luego lo referente al cuidado y desarrollo de potencialidades, y por último el respeto al cuerpo y la visión y proyección social. Por el contrario: por la difusión e interés genérico del cuerpo en la escuela, permite que sea abordado desde varios frentes, buscando que la concepción de imagen corporal no dependa únicamente de lo que la sociedad impone, sino que sea una herramienta de andamiaje en la ruta de aprendizaje y de conocimiento social.

\footnotetext{
7 Este requerimiento no quiere traducir exposición o burla. Es en la moderación donde se consigue ganar confianza por uno mismo y por los demás. Se consigue respeto propio y colectivo. Más allá sería mostrar un destacamento excesivo o figurar.
} 


\section{Reflexiones finales}

Las intenciones fisiológicas corpóreas constituyen sólo un ápice del objeto educativo y social del cuerpo humano. La imagen corporal se concibe como algo más que reconocer una belleza en términos estéticos, es un entramado donde se movilizan aspectos sociales, emocionales y sexuales. Por un lado, la imagen corporal determina una posición integral e individual frente a un común denominador de contexto que cambia según los protagonistas, la época y el lugar, aceptando o no la norma que prevalece. Por otro, establece un sutil equilibrio entre la provocación, el deseo y la búsqueda identitaria, que recree un código social que permita aproximarse a la cultura a la que se pertenece, sin alejarse de sus bases religiosas, sociales y políticas.

La imagen corporal se erige como un símbolo o una muestra de lo natural, lo propio, que, aunque cargado de influencias externas, recapitula elementos que encuentra en el contexto y luego los apropia para sí, y, aunque la imagen corporal suele aparecer en oposición bipolar logrando que un individuo entre en contradicción entre lo que es y lo que la sociedad le exige, su naturaleza no sugiere inclinar la balanza hacia uno de los dos lados, sino generar un punto de equilibrio que le permita defender y legitimar su cuerpo y su pensamiento.

\section{Referencias}

Barbero, J. (2001). La cultura de consumo, el cuerpo y la Educación Física. Departamento de Didáctica de la expresión Musical, Plástica y Corporal. Valladolid: Facultad de Educación, Universidad de Valladolid.

Bordieu, P. (1986). Notas provisionales sobre la percepción social del cuerpo. En Wright, et al., Materiales de Sociología Crítica. Madrid: La piqueta, 183-194.

Denis, D. (1994). El cuerpo enseñado. Barcelona: Paidós.

Elias, N. (1986). La génesis del deporte como problema sociológico en El deporte y el ocio en la civilización. México: FCE, 157-184.

Focault, M. (1978). Sexo, poder y gobierno de la identidad. Fragmento de Entrevista.

Focault, M. (1978). Cuerpos Dóciles en Vigilar y castigar. Madrid: Siglo XXI, 139-174.
Lapierre, A. (1990). El papel del cuerpo en la Educación. Boletín de Información y documentación Unisport, 11, 9-16.

Pedraz, M.V. (2010). Nómadas. Revista Crítica de Ciencias Sociales y Jurídicas, 28, 204.

Rodin, J., Silberstein, L., y Striegel-Moore, R. (1985). Women and weight: a normative discontent. En T. B. (Ed.). Psychology and Gender: NebraskaSymposium on Motivation). Lincoln: University of Nebraska Press, 267-307.

Schilder, P. (1950). The image and appearance of the human body. New York: International Universities Press.

Tubert, S. (2001). Desórdenes del cuerpo. Revista de Occidente, 134-135, 137-153. 


\section{Diálogo del conocimiento}

El interesante artículo sobre el papel que juega la sociedad y la educación en la construcción de la imagen corporal en las personas, que presenta la profesora Marly Stella Pérez, en su Artículo Cuerpo, escuela y sociedad, en la construcción de imagen corporal, invita a la reflexión sobre los modos en que los distintos grupos socioculturales han configurado simbólicamente la noción de la imagen corporal, como también, el papel que juega el docente en el afianzamiento o reafirmación de las identidades propias.

El texto resulta ser atractivo y sugestivo para el lector, quien necesariamente se ve obligado a centrar su mirada en sí mismo y en su sociedad. Lo relevante del tema, es la manera tan ágil de la autora, para mostrar las complejidades que determinan lo constitutivo en el ser humano, puesto que su estudio en cualquier óptica o disciplina debe abarcarse desde una mirada holística en su integralidad y ella lo hace muy bien.

La profesora Marly Stella Pérez, estructura el texto, con tres temas centrales y de mayor relevancia e implicados en la construcción de la imagen corporal de las personas, así: en primera instancia, se abarcan tópicos relacionados con la comprensión de la noción de imagen corporal desde diferentes ópticas, desde los distintos contextos y desde la historia sociocultural, identificando las distintas formas en que se filtra el concepto del cuerpo en la escuela. En segundo lugar, se abordan los aprendizajes en el aula, las vivencias y las experiencias de la escuela, implicados en la construcción de la imagen corpórea. Por último, se establece a manera de reflexiones, la importancia comprensiva de las dinámicas fluctuantes, que se dan entre el cuerpo, la escuela y sociedad, determinantes en construcción de la imagen corporal.

El texto podría profundizar el tema relacionado con la construcción de la imagen corporal desde la dimensión sociocultural, en los aspectos planteados por la autora, considero necesario que se complemente o se amplíe la perspectiva antropológica, sociológica e histórica, además del tema sobre el análisis del cuerpo, relacionado con la identidad del hombre y su percepción del mundo simbólico donde se inscribe la existencia del hombre corpóreo.

Abordar el tema de la percepción y valoraciones del cuerpo desde dimensiones socioculturales es relevante, plantearlo distinguiendo los dos modos o maneras de percibir y asumir el mundo y el universo del cuerpo. Por un lado, desde la lógica dualista-positivista que aún reina en la sociedad occidental y, por otro, las distintas percepciónes del cuerpo que encarnan las sociedades tradicionales, en ellas, el cuerpo no se divide ni se fragmenta, el cuerpo se integra o se fusiona con el universo o el cosmos. Profundizar un poco, en estas dos concepciones de mundo, permite al lector. Primero, ubicarse como perteneciente a una de estas dos culturas. Segundo, tener otros referentes que le permita cuestionar su propia cultura, y tercero, tener elementos claros, que le permita transformar su pensamiento, sea valorando o transformando sus propias concepciones de mundos posibles.

Hacernos conscientes de nuestras identidades culturales, es entender el mundo sociocultural al que pertenecemos, es poder reflexionar sobre nuestra propia cultura, sobre nuestra historia, cargada de las buenas o malas vivencias o experiencias que nos han construido como seres senti-pensantes, emocionales y soñadores. Es por ello, que la educación es la responsable como dinamizadora de la cultura, en la buena o mala formación de las personas. Es la responsable en hacer que hombres y mujeres sean felices o no, sean consientes de sus propias realidades socioculturales y tener las capacidades para cambiar su calidad de vida y la de los demás.

Isabel Cristina Muñoz Penagos 